\title{
SUBMANIFOLDS OF A RIEMANNIAN \\ MANIFOLD WITH \\ SEMISYMMETRIC METRIC CONNECTIONS
}

\begin{abstract}
ZENSHO NAKAO
Abstract. We derive the Gauss curvature equation and the CodazziMainardi equation with respect to a semisymmetric metric connection on a Riemannian manifold and the induced one on a submanifold. We then generalize the theorema egregium of Gauss.
\end{abstract}

1. Introduction. K. Yano [6] proved that a Riemannian manifold admits a semisymmetric metric connection with vanishing curvature tensor if and only if the manifold is conformally flat. Later, T. Imai [3], [4] studied some properties of hypersurfaces of a Riemannian manifold with a semisymmetric metric connection, and also obtained the Gauss curvature equation and the Codazzi-Mainardi equation with respect to a semisymmetric metric connection on a Riemannian manifold and the induced one on a hypersurface. The object of this paper is to derive the above two equations with respect to a semisymmetric metric connection on an $(n+p)$-dimensional Riemannian manifold and the induced one on an $n$-dimensional submanifold, and also to generalize the theorema egregium of Gauss. The notation of [5] will be used for the most part.

2. Gauss equation and Weingarten equation. Let $M$ be an $n$-dimensional Riemannian manifold isometrically imbedded in an $(n+p)$-dimensional Riemannian manifold $M^{\prime}$. We denote by $g$ the Riemannian metric tensor on $M^{\prime}$ as well as the induced one on $M$. Since $M$ has codimension $p$ we can locally choose $p$ cross sections $\xi_{i}, \quad i=1,2, \ldots, p$, of the normal bundle $T(M)^{\perp}$ of $M$ in $M^{\prime}$ which are orthonormal at each point of $M$.

A linear connection $\nabla^{\prime}$ on $M^{\prime}$ is called a semisymmetric metric connection if $\stackrel{\circ}{\nabla}^{\prime} g=0$ (metric) and the torsion tensor $\stackrel{\circ}{T}^{\prime}$ of $\stackrel{\circ}{\nabla}^{\prime}$ satisfies $\stackrel{\circ}{T}^{\prime}\left(X^{\prime}, Y^{\prime}\right)$ $=\pi\left(Y^{\prime}\right) X^{\prime}-\pi\left(X^{\prime}\right) Y^{\prime}$ (semisymmetric) for $X^{\prime}, Y^{\prime} \in \mathcal{X}\left(M^{\prime}\right)$, where $\pi$ is a 1 form on $M^{\prime}[6]$.

We now assume that a semisymmetric metric connection $\nabla^{\prime}$ is given on $M^{\prime}$ by

$$
\stackrel{\circ}{\nabla}_{X^{\prime}}^{\prime} Y^{\prime}=\nabla_{X^{\prime}}^{\prime} Y^{\prime}+\pi\left(Y^{\prime}\right) X^{\prime}-g\left(X^{\prime}, Y^{\prime}\right) P^{\prime}
$$

for $X^{\prime}, Y^{\prime} \in \mathcal{X}\left(M^{\prime}\right)$, where $\nabla^{\prime}$ denotes the Riemannian connection with respect to $g$ and $P^{\prime}$ a vector field on $M^{\prime}$ defined by $g\left(P^{\prime}, X^{\prime}\right)=\pi\left(X^{\prime}\right)$ for $X^{\prime}$

Received by the editors August 27, 1973 and, in revised form, December 30, 1974.

AMS (MOS) subject classifications (1970). Primary 53B15, 53B25; Secondary 53C05.

Key words and phrases. Codazzi-Mainardi equation, conformally flat space, Gauss curvature equation, mean curvature normal, sectional curvature, semisymmetric metric connection, totally umbilical submanifold. "

(c) American Mathematical Society 1976 
$\in \mathcal{X}\left(M^{\prime}\right)$ [6]. On $M$ we define a vector field $P$ and real-valued functions $\lambda_{i}, i=1,2, \ldots, p$, by decomposing $P^{\prime}$ into its unique tangential and normal components, thus

$$
P^{\prime}=P+\sum_{i=1}^{p} \lambda_{i} \xi_{i}
$$

If we denote by $\nabla$ the induced Riemannian connection on $M$ from $\nabla^{\prime}$ on $M^{\prime}$, then we have the Gauss equation (with respect to $\nabla^{\prime}$ ),

$$
\nabla_{X}^{\prime} Y=\nabla_{X} Y+\sum_{i=1}^{p} h_{i}(X, Y) \xi_{i}
$$

for $X, Y \in \mathscr{X}(M)$, where $h_{i}$ are the second fundamental forms on $M[5, \mathrm{pp}$. 10-21]. Let a connection $\nabla^{\circ}$ on $M$ be induced from the semisymmetric metric connection $\nabla^{\prime}$ on $M^{\prime}$ by the equation which may be called the Gauss equation with respect to $\stackrel{\circ}{\nabla}^{\prime}$,

$$
\stackrel{\circ}{\nabla}_{X}^{\prime} Y=\stackrel{\circ}{\nabla}_{X} Y+\sum_{i=1}^{p} \stackrel{\circ}{h}_{i}(X, Y) \xi_{i}
$$

for $X, Y \in \mathcal{X}(M)$, where $\stackrel{\circ}{h}_{i}$ are tensors of type $(0,2)$ on $M$.

From (1), using (2), (3) and (4), we obtain

$$
\begin{aligned}
\stackrel{\circ}{\nabla}_{X} Y+\sum_{i=1}^{p} \stackrel{\circ}{h}_{i}(X, Y) \xi_{i}= & \nabla_{X} Y+\sum_{i=1}^{p} h_{i}(X, Y) \xi_{i} \\
& +\pi(Y) X-g(X, Y)\left(P+\sum_{i=1}^{p} \lambda_{i} \xi_{i}\right)
\end{aligned}
$$

from which we get

$$
\stackrel{\circ}{\nabla}_{X} Y=\nabla_{X} Y+\pi(Y) X-g(X, Y) P
$$

for $X, Y \in \mathcal{X}(M)$, and we also have

$$
\check{h}_{i}=h_{i}-\lambda_{i} g \text {. }
$$

Using (6), we get $\stackrel{\circ}{\nabla}_{X}\{g(Y, Z)\}=\left(\stackrel{\circ}{\nabla}_{X} g\right)(Y, Z)+\nabla_{X}\{g(Y, Z)\}$, from which follows $\left(\stackrel{\circ}{\nabla}_{X} g\right)(Y, Z)=0$ for $X, Y, Z \in \mathcal{Q}(M)$, i.e.,

$$
\stackrel{\circ}{\nabla} g=0,
$$

and

$$
\stackrel{\circ}{T}(X, Y)=T(X, Y)+\pi(Y) X-\pi(X) Y=\pi(Y) X-\pi(X) Y
$$

for $X, Y \in \mathcal{X}(M)$, where $\stackrel{\circ}{T}$ and $T$ denote the torsion tensors of connections $\stackrel{\circ}{\nabla}$ and $\nabla$, respectively. Then from (8) and (9) we have [4]

THEOREM 1. The induced connection on a submanifold of a Riemannian manifold with a semisymmetric metric connection is also a semisymmetric metric connection. 
The Weingarten equation (with respect to $\nabla^{\prime}$ ) is given by

$$
\nabla_{X}^{\prime} \xi_{i}=-A_{i}(X)+D_{X} \xi_{i}
$$

for $X \in \mathcal{X}(M)$, where $A_{i}$ are tensors of type $(1,1)$ on $M$ and $D$ is a (metric) connection in the normal bundle $T(M)^{\perp}$ with respect to the fibre metric induced from $g$ [5, pp. 10-21].

Note that from (3) and (10) we have $h_{i}(X, Y)=g\left(Y, A_{i}(X)\right)$, and thus we get

$$
h_{i}(X, Y)=g\left(X, A_{i}(Y)\right)=g\left(A_{i}(X), Y\right)
$$

for $X, Y \in \mathfrak{X}(M)$ since $h_{i}$ are symmetric. While from (1) and (2) we have $\stackrel{\circ}{\nabla}_{X}^{\prime} \xi_{i}=\nabla_{X}^{\prime} \xi_{i}+\lambda_{i} X$, which together with (10) implies ${\stackrel{\circ}{\nabla^{\prime}}}_{X}^{\prime} \xi_{i}=-\left(A_{i}-\lambda_{i} I\right)$ $\cdot(X)+D_{X} \xi_{i}$, where $I$ is the identity tensor. Defining tensors $\AA_{i}$ of type $(1,1)$ on $M$ by $A_{i}=A_{i}-\lambda_{i} I$, we get a more concise expression,

$$
\stackrel{\circ}{\nabla}_{X}^{\prime} \xi_{i}=-\AA_{A}(X)+D_{X} \xi_{i}
$$

for $X \in \mathfrak{X}(M)$, which may be called the Weingarten equation with respect to $\stackrel{\circ}{\nabla}^{\prime}$.

We obtain, using (7) and (11), the following result which will be used later [1], [5, pp. 10-21]:

Lemma. The induced linear transformations, also denoted by $A_{i}$ and $\AA_{i}$, of the tangent space $T_{m}(M)$ at $m \in M$ defined by (10) and (12) satisfy, respectively, $h_{i}(X, Y)=g\left(A_{i}(X), Y\right)$ and $\stackrel{\circ}{h}_{i}(X, Y)=g\left(\AA_{i}(X), Y\right)$ for $X, Y \in T_{m}(M)$, and thus are symmetric with respect to $g$, i.e., $g\left(A_{i}(X), Y\right)=g\left(X, A_{i}(Y)\right)$ and $g\left(\stackrel{\AA}{A}_{i}(X), Y\right)=g\left(X, \stackrel{\AA}{A}_{i}(Y)\right)$ for $X, Y \in T_{m}(M)$.

The mean curvature normal $H$ of $M$ (with respect to $\nabla$ ) is given by $H=(1 / n) \sum_{i=1}^{p}\left(\operatorname{trace} A_{i}\right) \xi_{i}$ [5, pp. 29-42]. We define similarly the mean curvature normal $\stackrel{H}{H}$ of $M$ with respect to $\stackrel{\circ}{ }$ by $\stackrel{H}{H}=(1 / n) \sum_{i=1}^{p}\left(\right.$ trace $\left.\AA_{i}\right) \xi_{i}$. Let $X_{j}, j=1,2, \ldots, n$, be $n$ orthonormal local vector fields on $M$. Then $H$ and $H$ can be expressed as

$$
H=\frac{1}{n} \sum_{i=1}^{p}\left\{\sum_{j=1}^{n} h_{i}\left(X_{j}, X_{j}\right)\right\} \xi_{i}, \quad \stackrel{\circ}{H}=\frac{1}{n} \sum_{i=1}^{p}\left\{\sum_{j=1}^{n} \stackrel{\circ}{h}_{i}\left(X_{j}, X_{j}\right)\right\} \xi_{i} .
$$

If $h_{i}=k_{i} g$, where $k_{i}$ are real-valued functions on $M$, then $M$ is said to be totally umbilical (with respect to $\nabla$ ). Similarly, if $\check{h}_{i}=k_{i} g$, then $M$ is said to be totally umbilical with respect to $\nabla$ [4], [7, pp. 91-93].

We get from (2), (7) and (13) the following results [4]:

THEOREM 2. The mean curvature normal of $M$ and that of $M$ with respect to

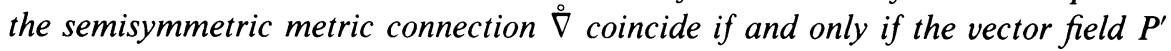
is tangent to $M$.

THEOREM 3. A submanifold $M$ of a Riemannian manifold $M^{\prime}$ is totally umbilical if and only if it is totally umbilical with respect to the semisymmetric metric connection $\stackrel{\circ}{\nabla}$. 
3. Gauss curvature equation and Codazzi-Mainardi equation. We denote by $R^{\prime}\left(X^{\prime}, Y^{\prime}\right) Z^{\prime}=\nabla_{X^{\prime}}^{\prime} \nabla_{Y^{\prime}}^{\prime} Z^{\prime}-\nabla_{Y^{\prime}}^{\prime} \nabla_{X^{\prime}}^{\prime} Z^{\prime}-\nabla_{\left[X^{\prime}, Y^{\prime}\right]}^{\prime} Z^{\prime} \quad$ for . $X^{\prime}, Y^{\prime}, Z^{\prime}$ $\in \mathcal{X}\left(M^{\prime}\right)$ and $R(X, Y) Z=\nabla_{X} \nabla_{Y} Z-\nabla_{Y} \nabla_{X} Z-\nabla_{[X, Y]} Z$ for $X, Y, Z$ $\in \mathcal{X}(M)$ the curvature tensors of $\nabla^{\prime}$ and $\nabla$, respectively. Then the Gauss curvature equation (with respect to $\nabla^{\prime}$ and $\nabla$ ) is given by

$$
\begin{aligned}
R^{\prime}(W, Z, X, Y)= & R(W, Z, X, Y) \\
& +\sum_{i=1}^{p}\left\{h_{i}(X, Z) h_{i}(Y, W)-h_{i}(Y, Z) h_{i}(X, W)\right\}
\end{aligned}
$$

for $X, Y, Z, W \in \mathfrak{X}(M)$, where

$$
R^{\prime}\left(W^{\prime}, Z^{\prime}, X^{\prime}, Y^{\prime}\right)=g\left(R^{\prime}\left(X^{\prime}, Y^{\prime}\right) Z^{\prime}, W^{\prime}\right)
$$

for $X^{\prime}, Y^{\prime}, Z^{\prime}, W^{\prime} \in \mathcal{X}\left(M^{\prime}\right)$ and

$$
R(W, Z, X, Y)=g(R(X, Y) Z, W)
$$

for $X, Y, Z, W \in \mathcal{X}(M)$ are, respectively, the Riemann-Christoffel curvature tensors of $M^{\prime}$ and $M$ (with respect to $\nabla^{\prime}$ and $\nabla$ ), and the Codazzi-Mainardi equation (with respect to $\nabla^{\prime}$ and $\nabla$ ) is given by

$$
\begin{aligned}
R^{\prime}\left(\xi_{i}, Z, X, Y\right)= & \left(\nabla_{X} h_{i}\right)(Y, Z)-\left(\nabla_{Y} h_{i}\right)(X, Z) \\
& +\sum_{j=1}^{p} g\left(\left\{h_{j}(Y, Z) D_{X} \xi_{j}-h_{j}(X, Z) D_{Y} \xi_{j}\right\}, \xi_{i}\right)
\end{aligned}
$$

for $X, Y, Z \in \mathcal{X}(M)[1],[5$, pp. 22-29].

Next we shall find the Gauss curvature equation and the Codazzi-Mainardi equation with respect to the semisymmetric metric connections $\dot{\circ}^{\prime}$ and $\stackrel{\circ}{\nabla}$. The curvature tensors of $\stackrel{\circ}{\nabla}^{\prime}$ and $\stackrel{\circ}{\nabla}^{\circ}$ are defined, respectively, by

$$
\stackrel{\circ}{R}^{\prime}\left(X^{\prime}, Y^{\prime}\right) Z^{\prime}=\stackrel{\circ}{\nabla}_{X^{\prime}}^{\prime} \stackrel{\circ}{\nabla}_{Y^{\prime}}^{\prime} Z^{\prime}-\stackrel{\circ}{\nabla}_{Y^{\prime}}^{\prime} \stackrel{\circ}{\nabla}_{X^{\prime}}^{\prime} Z^{\prime}-\stackrel{\circ}{\nabla}_{\left[X^{\prime}, Y^{\prime}\right]}^{\prime} Z^{\prime}
$$

for $X^{\prime}, Y^{\prime}, Z^{\prime} \in \mathcal{X}\left(M^{\prime}\right)$ and $\stackrel{\circ}{R}(X, Y) Z=\stackrel{\circ}{\nabla}_{X} \stackrel{\circ}{\nabla}_{Y} Z-\stackrel{\circ}{\nabla}_{Y} \stackrel{\circ}{\nabla}_{X} Z-\stackrel{\circ}{\nabla}_{[X, Y]} Z$ for $X, Y, Z \in X(M)$. Then from (4), (9) and (12) we get

$$
\begin{aligned}
\stackrel{\circ}{R}^{\prime}(X, Y) Z= & \stackrel{\circ}{R}(X, Y) Z+\sum_{i=1}^{p}\left\{\stackrel{\circ}{h}_{i}(X, Z) \stackrel{\circ}{A}_{i}(Y)-\stackrel{\circ}{h}_{i}(Y, Z) \stackrel{\circ}{A}_{i}(X)\right\} \\
& +\sum_{i=1}^{p}\left\{\left(\stackrel{\circ}{\nabla}_{X} \stackrel{\circ}{h}_{i}\right)(Y, Z)-\left(\stackrel{\circ}{\nabla}_{Y} \stackrel{\circ}{h}_{i}\right)(X, Z)\right. \\
& \left.+\stackrel{\circ}{h}_{i}(\pi(Y) X-\pi(X) Y, Z)\right\} \xi_{i} \\
& +\sum_{i=1}^{p}\left\{\stackrel{\circ}{h}_{i}(Y, Z) D_{X} \xi_{i}-\grave{h}_{i}(X, Z) D_{Y} \xi_{i}\right\}
\end{aligned}
$$

for $X, Y, Z \in \mathcal{X}(M)$.

We define the Riemann-Christoffel curvature tensors of $M^{\prime}$ and $M$ with respect to $\stackrel{\circ}{\nabla}^{\prime}$ and $\stackrel{\circ}{\nabla}$, respectively, by $R^{\prime}\left(W^{\prime}, Z^{\prime}, X^{\prime}, Y^{\prime}\right)=g\left(R^{\prime}\left(X^{\prime}, Y^{\prime}\right) Z^{\prime}, W^{\prime}\right)$ and $\stackrel{\circ}{R}(W, Z, X, Y)=g(\stackrel{R}{R}(X, Y) Z, W)$ for $X^{\prime}, Y^{\prime}, Z^{\prime}, W^{\prime} \in \mathcal{X}\left(M^{\prime}\right)$ and $X, Y$, $Z, W \in \mathfrak{X}(M)$. Then from (16) and the Lemma, we obtain the Gauss curvature equation with respect to $\stackrel{\circ}{\nabla}^{\prime}$ and $\stackrel{\circ}{\nabla}^{[}[4]$ : 


$$
\begin{aligned}
\stackrel{\circ}{R}^{\prime}(W, Z, X, Y)= & \stackrel{\circ}{R}(W, Z, X, Y) \\
& +\sum_{i=1}^{p}\left\{\stackrel{\circ}{h}_{1}(X, Z) \stackrel{\circ}{h}_{i}(Y, W)-\stackrel{\circ}{h}_{i}(Y, Z) \stackrel{\circ}{h}_{i}(X, W)\right\}
\end{aligned}
$$

for $X, Y, Z, W \in \mathfrak{X}(M)$. We also have from (16) the Codazzi-Mainardi equation with respect to $\dot{\nabla}^{\prime}$ and $\dot{\circ}^{\circ}[4]$ :

$$
\begin{aligned}
\stackrel{\circ}{R}^{\prime}\left(\xi_{i}, Z, X, Y\right)= & \left(\stackrel{\circ}{\nabla}_{X} \stackrel{\circ}{h}_{i}\right)(Y, Z)-\left(\stackrel{\circ}{\nabla}_{Y} \check{\circ}_{i}\right)(X, Z)+\stackrel{\circ}{h}_{i}(\pi(Y) X-\pi(X) Y, Z) \\
& +\sum_{j=1}^{p} g\left(\left\{\stackrel{\circ}{h}_{j}(Y, Z) D_{X} \xi_{j}-\stackrel{\circ}{h}_{j}(X, Z) D_{Y} \xi_{j}\right\}, \xi_{i}\right)
\end{aligned}
$$

for $X, Y, Z \in \mathscr{X}(M)$.

Now we suppose the Riemannian manifold $M^{\prime}$ is conformally flat and that the submanifold $M$ is totally umbilical, then we can assume $R^{\prime}=0$ [6], and we also have $\dot{h}_{i}=k_{i} g$, since $M$ is totally umbilical with respect to $\stackrel{\circ}{\nabla}$ by Theorem 3. Then from (17) we get

$$
\stackrel{\circ}{R}(W, Z, X, Y)=\{g(Y, Z) g(X, W)-g(X, Z) g(Y, W)\} \sum_{i=1}^{p}\left(k_{i}\right)^{2}
$$

for $X, Y, Z, W \in \mathfrak{X}(M)$, which implies that $M$ is also conformally flat $(n>$ 3) [3]. Thus we have [4]

THEOREM 4. A totally umbilical submanifold in a conformally flat Riemannian manifold is conformally flat.

4. Theorema egregium. We obtain a generalization of the theorema egregium of Gauss with respect to semisymmetric metric connection by the method of N. Hicks [1].

From the Gauss curvature equation (17) and the Lemma, we get

$$
\begin{aligned}
\AA^{\prime}(X, Y, X, Y)= & \stackrel{\circ}{R}(X, Y, X, Y) \\
& +\sum_{i=1}^{p}\left\{g\left(\stackrel{\circ}{A}_{i}(X), Y\right)^{2}-g\left(\stackrel{\circ}{A}_{i}(X), X\right) g\left(\stackrel{\circ}{A}_{i}(Y), Y\right)\right\}
\end{aligned}
$$

for $X, Y \in \mathscr{X}(M)$. Therefore we have

THEOREM 5. Let $\mathscr{P}$ be a 2-dimensional subspace of $T_{m}(M)$, and let $R^{\prime}(\mathscr{P})$ and $\AA^{\circ}(\mathscr{P})$ be, respectively, the sectional curvatures of $\mathscr{P}$ in $M^{\prime}$ and $M$ with respect to the semisymmetric connections $\stackrel{\circ}{ }^{\prime}$ and $\stackrel{\circ}{\nabla}^{\circ}$. If $X$ and $Y$ form an orthonormal base of $\mathcal{P}$, then

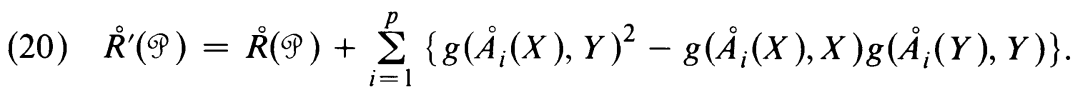

As immediate consequences of Theorem 5 we get [6]

Corollary 1. If $\operatorname{dim} M^{\prime}=3$ and $M$ is a surface in $M^{\prime}$, then the determinant of $\AA_{i}$ (where there is now only one such map) is independent of $\AA_{i}$ but depends only on the Riemannian metric tensor $g$ and the semisymmetric metric connections $\stackrel{\circ}{\nabla}^{\prime}$ and $\stackrel{\circ}{\nabla}$. 
COROLlary 2. If $M^{\prime}$ is a conformally flat Riemannian manifold of dimension 3 and $M$ is a surface in $M^{\prime}$, then there exists a semisymmetric metric connection $\stackrel{\circ}{\nabla}$ on $M$ for which det $\AA_{i}$ is an intrinsic invariant of $M$, and, when $P^{\prime}$ is tangent to $M$, det $\dot{A}_{i}(=\dot{R}(\mathcal{P}))$ is equal to $\operatorname{det} A_{i}$ which is the Gaussian curvature of $M$.

\section{REFERENCES}

1. N. J. Hicks, Submanifolds of semi-Riemannian manifolds, Rend. Circ. Mat. Palermo (2) 12 (1963), 137-149. MR 29 \#1601.

2. —- Notes on differential geometry, Van Nostrand Math. Studies, no. 3, Van Nostrand, Princeton, N. J., 1965. MR 31 \#3936.

3. T. Imai, Notes on semi-symmetric metric connections, Tensor 24 (1972), 293-296.

4. - Hypersurfaces of a Riemannian manifold with semi-symmetric metric connection, Tensor 23 (1972), 300-306.

5. S. Kobayashi and K. Nomizu, Foundations of differential geometry, Vol. II, Interscience Tracts in Pure and Appl. Math., no. 15, Vol. II, Interscience, New York, 1969. MR 38 \#6501.

6. K. Yano, On semi-symmetric metric connection, Rev. Roumaine Math. Pures Appl. 15 (1970), 1579-1586. MR 43 \# 1078.

7. - Integral formulas in Riemannian geometry, Pure and Appl. Math., no. 1, Dekker, New York, 1970. MR 44 \#2174.

Department of Mathematics, University of Oklahoma, Norman, Oklahoma 73069 